\title{
¿Es la tecnología blockchain compatible con la Economía Social y Solidaria? Hacia un nuevo paradigma
}

\author{
August Corrons Giménez \\ Marta Gil Ibáñez
}

RESUMEN: La significativa evolución que las tecnologías de la información han experimentado en los últimos años ha afectado a todos los sectores, incluido el de la Economía Social y Solidaria. El desarrollo emergente de la tecnología blockchain y sus posibilidades para modificar las formas de colaboración solidaria de la ciudadanía, presentan un reto actual y un cambio de paradigma para el Tercer Sector. El objeto del presente artículo es analizar las posibilidades existentes de que ambos ámbitos compartan valores, principios y objetivos. Para ello, en primer lugar y como punto de referencia, se presentan los conceptos de Economía Social y Solidaria y de blockchain. A continuación, se presentan las compatibilidades de ambos ámbitos y se relacionan sus componentes. Por último, el artículo finaliza con un apartado de conclusiones y futuras líneas de investigación.

PALABRAS CLAVE: Economía social y solidaria, blockchain, tecnología, gobernanza, valores.

CLAVES ECONLIT: A13, E5, F3, L3, O3.

Cómo citar este artículo / How to cite this article: CORRONS, A. \& GIL, M. (2019): "¿Es la tecnología blockchain compatible con la Economía Social y Solidaria? Hacia un nuevo paradigma", CIRIEC-España, Revista de Economía Pública, Social y Cooperativa, 95, 191-215. DOI: 10.7203/CIRIEC-E.95.12984.

Correspondencia: August Corrons Giménez, Doctor en Desarrollo Local y Cooperación Internacional de la Universidad Jaume I, Profesor titular de la Universitat Oberta de Catalunya, y Marta Gil Ibáñez, Doctora en Economía Social de la Universitat de València, Profesora colaboradora de la Universitat Oberta de Catalunya. E-mail de contacto: mgilib@uoc.edu. 


\section{EXPANDED ABSTRACT}

\section{Is blockchain technology compatible with the Social and Solidarity Economy? Towards a new paradigm}

Spanish Law 5/2011, of 29 March, defines Social Economy as the set of economic and business activities that, in the private sphere, are carried out by entities that pursue the general economic or social interest, following the principles of the primacy of persons and the social purpose over capital, the promotion of internal solidarity and solidarity with society, and last, independence from public authorities. In other words, the Social and Solidarity Economy is a socio-economic area based on three main axes that determine its transforming character: democratic and participatory management, orientation towards human needs and commitment to the environment. In this way, it represents a new way of doing things, rather than a path towards a specific goal. In Spain, with the approval of the Social Economy Law in 2011, the sector has experienced a significant boost and growth in recent years.

At the same time, new technologies are representing a disruptive change in our current society and are modifying the way we relate to each other. At this point, it is important to differentiate between the Internet of information and the Internet of value. The first is the tool that enables the free circulation of information throughout the planet and has led to the creation of new business models such as Google, Facebook or Amazon, among others. As for the Internet of value, based precisely on blockchain technology, it is the tool that makes possible to share and manage the value of assets or goods in a digital and decentralized way, without the need to depend on a trusted entity that centralizes the process. Blockchain technology, released in 2009 by Bitcoin, is a decentralized database that is distributed among a multitude of different participants. This distribution system establishes a system of group trust, since the information is distributed among different agents, it cannot be altered. Blockchain will revolution the future development of economies and societies, with a potential capable of transforming both industry and services, as well as social relations. In this case, the initiative has started in the financial sector mainly, although as it is evident, its application in the future will not have to be exclusive to that sector.

The emergent development of blockchain technology and its possibilities to modify the forms of solidarity collaboration of citizens present a current challenge and a paradigm shift for the Third Sector. Today, society is changing the way to inform, to communicate and to relate. Faced with this reality, it is increasingly aware of its transforming role and driving agent of change.

The significant evolution that information technologies have undergone in recent years has affected all sectors, including the Social and Solidarity Economy. For this reason, the aim of this article is 
to analyze the combination of the concept of Social and Solidarity Economy and blockchain, analyzing the possibilities of both areas sharing values, principles and objectives. The Social and Solidarity Economy framework developed by Chaves and Monzón (2018), as well as Corrons, Fuster and Espelt (2017), is mainly used for this purpose. In the first place, the concept of Social and Solidarity Economy and its current framework are analyzed, highlighting its three main vertebral axes. On the other hand, and based on the research led by Preukschat on blockchain, in addition to the definition of the concept, the levels at which it is stratified are identified: structure, properties, functional characteristics and usability. In order to complete the study, an analysis of the socioeconomic implications and barriers presented by this technology is presented.

In short, the blockchain offers an opportunity to give authenticity to what is understood by collaborative with respect to the proposals that exist today. However, the main barriers that this technology presents are related to the implications of the current socioeconomic system. It provides freedom of action to individuals in the face of oligopolies, large corporations and States. The free exchange of crypto currencies implies no need for banks. The privacy of economic transactions implies the difficulty of taxation by States. And global and universal registration, without anyone being able to manipulate it, implies that all transactions are stored forever and, therefore, that there is no place for the right to forget or to a second chance.

At present, there is a lack of research and studies that deal with this new technology, its possibilities and barriers to adapt to the consolidated concept of Social Economy. In this way, this analysis focused mainly on the study of existing literature, shows how blockchain technology has become the new paradigm in the Third Sector and how it can be the basic tool for changing the behavior of citizens in solidarity issues.

In the near future, the disruptive technologies are called to cause a great impact in the traditional systems in benefit of the citizens, forcing to a vindication of the inefficient and obsolete current schemes. Here is where blockchain technology, configured in a way compatible with the principles of the Social Economy, can become a key tool that strengthens the Social Economy sector, that allows it to continue growing up and that manages to increase the impact of the aims of the entities that make it up.

KEYWORDS: Social and solidarity economy, blockchain, technology, governance, values. 


\section{Introducción}

La Economía Social y Solidaria es un ámbito socioeconómico basado en tres grandes ejes vertebradores que determinan su carácter transformador: la gestión democrática y participativa, la orientación a las necesidades humanas y el compromiso con el entorno. De esta forma, supone una nueva forma de hacer las cosas, más que un camino rumbo a un fin determinado. En España, con la aprobación de la Ley 5/2011 de Economía Social, el sector ha experimentado un impulso y crecimiento significativo en los últimos años.

De forma paralela, las nuevas tecnologías están suponiendo un cambio disruptivo en nuestra sociedad actual y están modificando nuestra manera de relacionarnos. En este punto, es importante diferenciar entre el Internet de la información y el del valor. El primero es la herramienta que posibilita la libre circulación de información en todo el planeta y que ha propiciado la creación de nuevos modelos de negocio como por ejemplo Google, Facebook o Amazon, entre otros. En cuanto al Internet del valor, basado precisamente en la tecnología blockchain, representa la herramienta que posibilita compartir y gestionar el valor de activos o bienes de una forma digital y descentralizada, sin la necesidad de depender de una entidad de confianza que centralice el proceso. La tecnología blockchain, con fecha de aparición en el año 2009 de la mano del Bitcoin, es una base de datos descentralizada que está distribuida entre multitud de diferentes participantes. La blockchain es un ámbito desde el que se está revolucionando el desarrollo futuro de las economías y las sociedades, con un potencial capaz de transformar tanto la industria y los servicios, como las relaciones sociales. En este caso, la iniciativa de uso ha comenzado en el sector financiero principalmente, aunque como es evidente, su aplicación en el futuro no tendrá por qué ser exclusiva de dicho sector.

El desarrollo emergente de la tecnología blockchain y sus posibilidades para modificar las formas de colaboración solidaria de la ciudadanía, presentan un reto actual y un cambio de paradigma para el Tercer Sector. En la actualidad, la sociedad está cambiando el modo en el que se informa, se comunica y se relaciona. Frente a esta realidad, es cada vez más consciente de su papel transformador y agente impulsor de cambio.

La significativa evolución que las tecnologías de la información han experimentado en los últimos años ha afectado a todos los sectores, incluido el de la Economía Social y Solidaria. Por ello, el objeto del presente artículo es analizar la combinación del concepto de Economía Social y Solidaria y de blockchain, analizando las posibilidades de que ambos ámbitos compartan valores, principios y objetivos. A tal efecto, se utiliza principalmente el marco de Economía Social y Solidaria desarrollado por Chaves y Monzón (2018), así como por Corrons, Fuster y Espelt (2017). En primer lugar, se analiza el concepto de Economía Social y Solidaria y su marco actual, destacando sus tres grandes ejes 
vertebradores. Por otro lado y teniendo como base la investigación liderada por Preukschat sobre blockchain, se identifican además de la definición del concepto, los niveles en los que se estratifica la misma: estructura, propiedades, características funcionales y usabilidad. Para completar el estudio se realiza un análisis de las implicaciones socioecónomicas y barreras que presenta dicha tecnología.

Actualmente, se carece de investigaciones y estudios que traten desde una perspectiva de comparación esta nueva corriente tecnológica, sus posibilidades y barreras para adaptarse con el sí consolidado concepto de Economía Social. De esta forma, este análisis centrado principalmente en el estudio de la literatura existente, demuestra la forma en la que la tecnología blockchain se ha convertido en el nuevo paradigma en el Tercer Sector y cómo puede suponer la herramienta base del cambio de comportamiento de la ciudadanía en temas solidarios.

\section{La Economía Social y Solidaria}

\subsection{Introducción}

En este apartado se procede a la presentación y concepción de lo que es y representa la Economía Social y Solidaria. Para ello, se parte del enfoque y el contenido desarrollado por Corrons, Fuster y Espelt (2017) en el trabajo titulado "Economia Social i Solidària a l'Àrea Metropolitana de Barcelona: contextualització i situació actual". Todo ello, junto con otra serie de aportaciones referenciadas a lo largo del texto y/o especificadas en el apartado 5 de bibliografía y referencias consultadas.

Según el libro "La economía solidaria en 100 palabras" de Jordi García (2017), la Economía Social y Solidaria es el conjunto de prácticas socioeconómicas, formales o informales, colectivas pero también individuales, que priorizan la satisfacción de las necesidades y las aspiraciones de sus miembros y/u otras personas por encima del lucro; cuando son colectivas, la propiedad también lo es, y la gestión es democrática; son independientes respecto a cualquier entidad pública o privada, actúan orientadas por los valores de equidad, solidaridad, sostenibilidad, participación, inclusión y compromiso con la comunidad, y son promotoras de cambio social. Tal y como concluyen diversos autores (Gómez y Gómez-Álvarez, 2016; Chaves y Monzón, 2018), en la actualidad el concepto de Economía Social y el de Nonprofit Organization, continúan bajo el paraguas del espacio existente entre la economía pública y capitalista.

Un punto de inflexión importante en España y que ha impulsado el desarrollo del sector los últimos años ha sido la aprobación de la Ley 5/2011, de 29 de marzo, de Economía Social (BOE 76, de 
30 de marzo de 2011). Además, con la reciente aprobación de la Ley 31/2015, de 9 de septiembre, se sigue reforzando estableciendo medidas de desarrollo y fomento de la Economía Social en España. Según el propio texto de la primera, se entiende por Economía Social al conjunto de actividades económicas y empresariales, que en el ámbito privado llevan a cabo aquellas entidades que, de conformidad con los siguientes principios, persiguen el interés general económico o social, 0 ambos:

- Primacía de las personas y del fin social sobre el capital, que se concreta en gestión autónoma y transparente, democrática y participativa, que lleva a priorizar la toma de decisiones más en función de las personas y sus aportaciones de trabajo y servicios prestados a la entidad 0 en función del fin social, que en relación a sus aportaciones al capital social.

- Aplicación de los resultados obtenidos de la actividad económica principalmente en función del trabajo aportado y servicio o actividad realizada por las socias y socios o por sus miembros y, en su caso, al fin social objeto de la entidad.

- Promoción de la solidaridad interna y con la sociedad que favorezca el compromiso con el desarrollo local, la igualdad de oportunidades entre hombres y mujeres, la cohesión social, la inserción de personas en riesgo de exclusión social, la generación de empleo estable y de calidad, la conciliación de la vida personal, familiar y laboral y la sostenibilidad.

- Independencia respecto a los poderes públicos.

La Economía Social y Solidaria representa un conjunto de movimientos y fenómenos socioeconómicos que, en contraposición al sistema económico capitalista, no persiguen otra cosa que el logro del objetivo inicial y fundamental de la economía: administrar recursos limitados para satisfacer las necesidades de todos y cada uno de los seres humanos. Una economía ya existente, cada vez más relevante, que ya no representa una alternativa al sistema actual sino una realidad a la que sumarse en pro de todos y del medio ambiente. Al igual que sucede en el caso de la banca ética, donde a priori no habría que añadir el apellido "ética" a una banca que tendría que serlo por defecto, en el caso de la Economía Social y Solidaria no habría que añadir la etiqueta "social y solidaria", pues a priori y según el significado propio de la palabra economía, esta tendría que ser social y solidaria por defecto. No obstante, esto no sucede así ni en el caso de la banca ni en el caso de la economía. Tal es así, que se sigue considerando más una forma de vivir que un camino a un fin determinado (Bretos y Morandeira, 2016; Gómez y Gómez-Álvarez, 2016; Chaves y Zimmer, 2017). Por lo tanto, se continúa añadiendo palabras que complementan estos conceptos y aportan una información adicional que les permite caracterizar y diferenciarse del resto, a la espera de que el tiempo las vaya aproximando e integrando.

\subsection{Concepción global del sistema socioeconómico vigente}

A continuación se presenta en la llustración 1, un esquema conceptual que plasma de forma global el sistema socioeconómico vigente, subdividiéndolo en diferentes polos, ámbitos y ejes vertebradores. 


\section{llustración 1. Concepción global del sistema socioeconómico vigente}

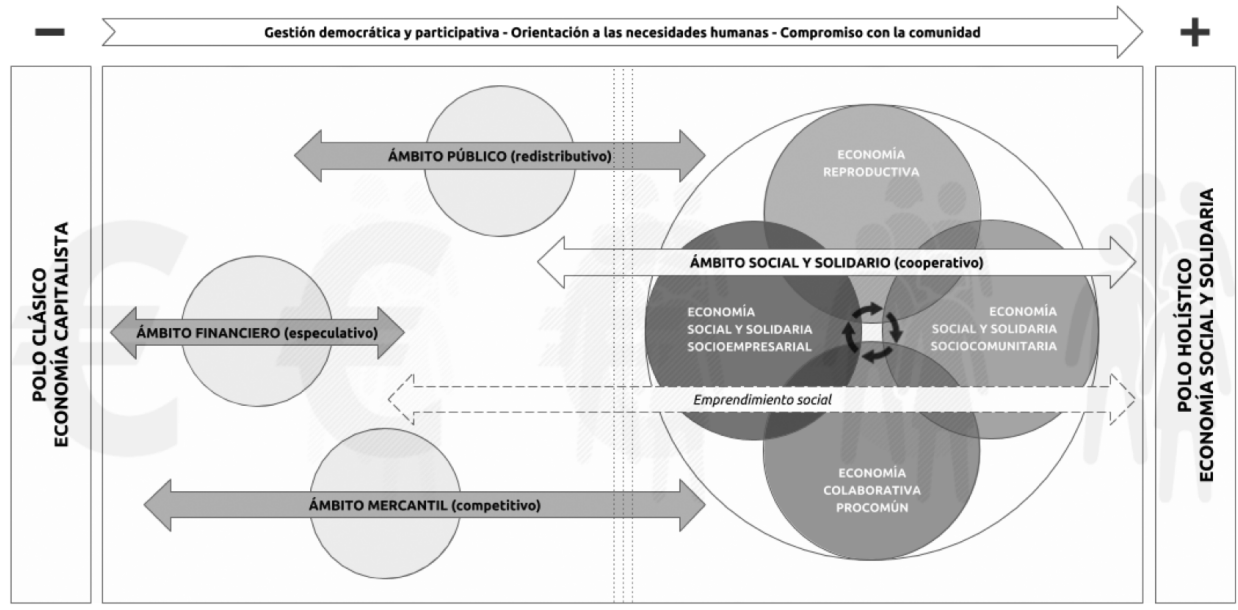

FUENTE: Economia Social i Solidària a l'Àrea Metropolitana de Barcelona: contextualització i situació actual. Corrons, Fuster y Espelt (2017).

Tal y como se aprecia en la ilustración anterior, el esquema representa dos polos claramente opuestos:

- Polo clásico. Representa un paradigma mecanicista y lineal, asimilable a la economía capitalista. Está caracterizado por el poder concentrado y jerárquico, por la sostenibilidad parcial o económica, y por la búsqueda de motivaciones extrínsecas.

- Polo holístico. Representa un paradigma orgánico y complejo, asimilable a la Economía Social y Solidaria. Está caracterizado por el poder distribuido, por la sostenibilidad integral y por la búsqueda de motivaciones intrínsecas.

Se trata de dos paradigmas llevados al extremo, es decir, en total contraposición el uno con el otro. No obstante, entre dos extremos opuestos hay un abanico infinito de posibilidades y combinaciones. Es precisamente dentro de este abanico de posibilidades en el que se emplazan los cuatro grandes ámbitos en los que se subdivide el sistema socioeconómico vigente:

- El ámbito público (redistributivo). Ámbito socioeconómico formado por el conjunto de actividades e iniciativas económicas promovidas desde la administración pública. Se trata de un ámbito situado en la mitad clásica del esquema, si bien es lo más cercano a la mitad holística, dada su función pública. Algunas de las prácticas que permiten la aproximación del ámbito 
público al ámbito social y solidario son la contratación pública responsable, la municipalización participativa y la empresa municipal.

- El ámbito mercantil (competitivo). Ámbito socioeconómico formado por las actividades promovidas desde la economía de mercado tradicional. Se trata de un ámbito emplazado en la mitad clásica del esquema, a medio camino entre los ámbitos público y financiero. Su recorrido de aproximación al ámbito social y solidario incluye, entre otros, prácticas como la economía colaborativa corporativa, la economía del bien común, la economía circular y la responsabilidad social corporativa.

- El ámbito financiero (especulativo). Ámbito socioeconómico hegemónico, en el sentido que ordena los ámbitos público y mercantil a través de mecanismos de deuda externa y fondos de inversión. Se trata de un ámbito situado en la mitad clásica del esquema, siendo el más alejado de la mitad holística. Y, por lo tanto, el más cercano al polo clásico asimilable a la economía capitalista. El recorrido de aproximación de este ámbito hacia el social y solidario es relativamente pequeño, estando básicamente limitado a las acciones de responsabilidad social corporativa que puedan llevar a cabo las entidades financieras.

- El ámbito social y solidario (cooperativo). Ámbito socioeconómico formado por el conjunto de actividades e iniciativas que se diferencian claramente del marco dominante de economía de mercado competitiva y con afán de lucro. Se trata de un ámbito emplazado en la mitad holística del esquema, abarcando toda su extensión en función de la tipología de iniciativas y prácticas que la representan. Actúa en todas las fases del ciclo económico: la gestión de los recursos, la producción, la comercialización, el consumo, el sistema financiero, la distribución del excedente y la circulación monetaria. Está caracterizado, en mayor o menor grado, por el poder distribuido y por la sostenibilidad integral, así como por la investigación de motivaciones intrínsecas. Se basa, en cualquier caso, en la gestión democrática y participativa, la orientación a las necesidades humanas y el compromiso con la comunidad.

Para emplazar estos cuatro grandes ámbitos dentro del esquema anterior, se tiene en consideración la alineación de cada uno de ellos con los tres grandes ejes vertebradores del ámbito cooperativo de la Economía Social y Solidaria:

- Gestión democrática y participativa. En lugar del modelo organizativo jerárquico donde unas cuántas personas o clases dirigentes controlan, gestionan y deciden sobre los recursos, el patrimonio, la información y el futuro de la mayoría, la Economía Social y Solidaria introduce la democracia en la economía y en la empresa.

- Orientación a las necesidades humanas. La Economía Social y Solidaria trata de recuperar la función originaria de la economía poniéndola al servicio de las personas para gestionar los recursos equitativamente y explotarlos de manera sostenible, así como crear un modo de producción que convierta el trabajo en un instrumento de satisfacción de las necesidades humanas. 
- Compromiso con la comunidad. Las organizaciones de la Economía Social y Solidaria contribuyen a la mejora de la sociedad, creando ocupación, prestando servicios, vinculándose al territorio, apoyando causas sociales, financiando iniciativas de solidaridad con los países empobrecidos y colaborando con movimientos sociales transformadores.

En este sentido, el polo clásico es el extremo totalmente opuesto a estas tres características, mientras que el polo holístico es en esencia, la gestión democrática y participativa, la orientación a las necesidades humanas y el compromiso con la comunidad.

Aparte de estos cuatro grandes ámbitos, se destaca el emprendimiento social como un tipo de iniciativa entre el ámbito mercantil (competitivo) y el ámbito social y solidario (cooperativo). La individualidad o colectividad del emprendimiento, así como su grado de gestión democrática y participativa, son las que determinan finalmente si el emprendimiento se aproxima más a uno 0 a otro ámbito.

A continuación se analiza la contextualización general de estos ámbitos, focalizando en el social y solidario y justificando el posicionamiento de todos y cada uno de ellos dentro del esquema. Se trata de un posicionamiento conceptual y cualitativo con el objetivo de identificar la posición relativa de cada uno de estos ámbitos, tanto entre ellos como en relación a los polos. Además, se analizan las herramientas que desde los ámbitos público, mercantil y financiero se están empleando o se pueden emplear para aproximarse, en mayor o menor grado, al trasfondo y a los valores del ámbito social y solidario.

\subsection{Confluencia de ámbitos y casos particulares}

Tal y como se puede apreciar en la ilustración anterior, el ámbito social y solidario (cooperativo) llega a cruzar la zona intermedia, adentrándose en la mitad clásica. Se evidencia este hecho para tener en consideración la particularidad de la Economía Social y Solidaria en su vertiente socio empresarial. Es decir, puede darse el caso de entidades con forma jurídica propia de la Economía Social y Solidaria (por ejemplo cooperativas) que, aun así, no tengan del todo integrado uno o varios ejes vertebradores de ésta, ya sea la gestión democrática y participativa, la orientación a las necesidades humanas o el compromiso con la comunidad. Lo mismo puede suceder en el caso del emprendimiento social.

En el caso del ámbito mercantil (especulativo) puede darse una situación similar pero al revés. Es decir, podría darse el caso de entidades con forma jurídica propia de la economía mercantil (por ejemplo sociedades limitadas) que, aun así, tengan del todo integrados los tres ejes vertebradores de la Economía Social y Solidaria. Es por este motivo que en el esquema presentado anteriormente se evidencia gráficamente cómo el ámbito mercantil llega a cruzar la zona intermedia, adentrándose en la mitad holística. Independientemente de esto, o como vía complementaria, hace falta también tener en consideración las prácticas anteriormente expuestas que permiten la aproximación del ámbito mercantil al social y solidario, como serían la economía colaborativa corporativa, la economía del bien común, la economía circular y la responsabilidad social corporativa. 
En el caso del ámbito público (redistributivo), su inmersión en la mitad holística o en el ámbito social y solidario tiene lugar como consecuencia de las prácticas de aproximación explicadas anteriormente, como por ejemplo la contratación y compra pública responsables, las municipalizaciones participativas y las empresas municipales.

Por último, y en el caso del ámbito financiero (especulativo), la aproximación que puede representar la práctica de la responsabilidad social corporativa no hace aproximarlo mucho al ámbito social y solidario, quedando muy lejos todavía de la mitad holística del esquema.

\section{La tecnología blockchain}

\subsection{Introducción}

El término blockchain se puede traducir literalmente como "cadena de bloques". Pero, ¿qué es y qué representa exactamente esta tecnología? ¿Cuál es la estructura que la conforma? ¿Y las propiedades de las que dispone? ¿Cuáles son las características funcionales de las diferentes tipologías existentes? ¿Y la usabilidad que se le puede dar?

Antes de dar respuesta a estas y a otras preguntas, se tiene que tener en cuenta que la reciente aparición de esta tecnología, datada inicialmente de enero del año 2009 de la mano del Bitcoin, pero con un boom que no ha tenido lugar hasta hace apenas un par de años, hace que apenas haya literatura escrita al respeto, sobre todo en lengua castellana, tanto a nivel académico como divulgativo. En cuanto a instituciones o iniciativas que hagan difusión e impulsen esta tecnología a nivel nacional, podemos destacar la comunidad Blockchain España y la asociación recientemente creada Blockchain Cataluña. Es precisamente desde la comunidad Blockchain España desde donde se ha publicado el libro "Blockchain: la revolución industrial de Internet", coordinado por Álex Preukschat, un referente en el ámbito de estudio. Para la presentación y concepción de lo que es y representa la tecnología blockchain en este apartado, se parte precisamente del enfoque y el contenido desarrollado en este libro. Se tiene en cuenta también la última publicación de Preukschat de 2018, la Comunidad Blockchain. Todo ello, junto con otra serie de aportaciones referenciadas a lo largo del texto y/o especificadas en el apartado 5 de bibliografía y referencias consultadas.

\subsection{Del Internet de la información al Internet del valor}

El Internet de la información es la herramienta que posibilita la libre circulación de la misma en todo el planeta, propiciando la creación de nuevos modelos de negocio como por ejemplo Google, 
Facebook o Amazon, entre otros. En cuanto al Internet del valor, basado precisamente en la tecnología blockchain, representa la herramienta que posibilita compartir y gestionar el valor de activos o bienes de una forma digital y descentralizada, sin la necesidad de depender de una entidad de confianza que centralice el proceso.

Al igual que el Internet de la información ha representado un cambio radical en nuestras vidas, creando oportunidades para promover modelos de negocio en nuevos sectores de la economía y cambiando las dinámicas de las relaciones sociales, el Internet del valor representa un próximo paso en la evolución natural de la red. Si Internet supuso en su momento una revolución en el acceso y la difusión de la información, la tecnología blockchain representa una revolución en la transmisión de valor a través de la propia red que representa Internet.

El Internet de la información empezó a tomar forma dentro de los sectores militar y académico y desde ellos se expandió al resto de industrias. En el Internet del valor ha sido el sector financiero el primero en tomar la iniciativa. No obstante, y al igual que en el caso anterior, la aplicación de la tecnología blockchain no será exclusiva de un único sector, más bien al contrario, pues cada vez son más los sectores que exploran todo su potencial. La tecnología blockchain tendrá un impacto directo en diferentes ámbitos, como por ejemplo la banca, los seguros, las telecomunicaciones, la energía, la industria 4.0, la salud, las pymes, el juego online, los medios, las ONGs, el sector público, etc.

\section{3. ¿Qué es y cómo funciona la blockchain?}

Una blockchain es una base de datos descentralizada, que no puede ser alterada y que se encuentra distribuida entre diferentes participantes, al mismo tiempo que está protegida criptográficamente y organizada en bloques de transacciones relacionados entre ellos de forma matemática. Es un sistema que permite que diferentes partes que no confían plenamente las unas en las otras puedan mantener un consenso sobre la existencia, el estado y la evolución de una serie de factores compartidos. Este consenso es precisamente la clave del sistema blockchain, puesto que es el fundamento que permite que todos los participantes puedan confiar en la información que se encuentra grabada en éste.

La blockchain agrupa transacciones, sean del tipo de que sean, en bloques que están encadenados entre ellos. Las transacciones, llevadas a cabo de usuario a usuario, están enlazadas las unas con las otras, es decir, encadenadas. Por lo tanto, no pueden separarse, de forma que el acceso a una determinada transacción permite conocer todo su historial previo. La blockchain utiliza técnicas criptográficas, hecho que permite firmar las transacciones pero que no puedan ser manipuladas, otorgando una fiabilidad completa sin la necesidad de ningún organismo certificador que pudiera perder los registros. Así mismo, los bloques que agrupan las diferentes transacciones son introducidos en un libro de registro global y universal, completamente descentralizado, que asegura que nadie sea el propietario, evitando así que nadie tenga el control absoluto ni los pueda manipular. A continuación el ejemplo de una transacción, el caso de la compra de un vehículo de segunda mano. Esta compra generará 
una nueva transacción, que será ligada a las transacciones anteriores asociadas a aquel mismo vehículo. Por lo tanto, éste tendrá un historial completo, de forma que el nuevo comprador podrá comprobar si el vehículo ha tenido algún tipo de reparación grave, a qué precio fue comprado, si ha tenido más de un propietario, o cualquier otro detalle registrado previamente como transacción.

La blockchain es un libro mayor distribuido que proporciona una manera para que la información sea registrada y compartida por parte de una comunidad. En esta comunidad, cada miembro mantiene su propia copia de la información, y todos los miembros tienen que validar colectivamente cualquier actualización. La información podría representar transacciones, contratos, activos, identidades, o prácticamente cualquier cosa que pueda ser descrita de manera digital. Los asentamientos son permanentes, transparentes y se pueden buscar, lo cual hace posible que los miembros de la comunidad vean las historias de la transacción. Cada actualización es un nuevo bloque adicionado al final de la cadena. Un protocolo administra las nuevas ediciones o cómo los nuevos asentamientos son iniciados, validados, registrados y distribuidos. La privacidad también puede ser selectivamente forzada, permitiendo grados diversos de anonimato o protección de información sensible más allá de a quien explícitamente se le haya dado acceso. Con blockchain, la criptografía reemplaza a los terceros intermediarios como guardián de la confianza, con todos los participantes en la blockchain para certificar la integridad del conjunto.

\subsection{Niveles en los que se estratifica una blockchain}

A continuación se analizan los cuatro niveles en los que se estratifica la tecnología blockchain: la estructura que la conforma, las propiedades de las que dispone, las características funcionales de las diferentes tipologías existentes, y la usabilidad que se le puede dar.

\subsubsection{Estructura que conforma una blockchain}

Para entender el alcance de la tecnología blockchain hay que conocer los elementos básicos de los que se compone su estructura:

- Nodo. Puede ser un ordenador personal o, según la complejidad de la red, una mega computadora. Independientemente de la capacidad de computación, todas los nodos tienen que poseer el mismo software o protocolo para poderse comunicar entre sí, ya sea en una red pública, privada o híbrida. Estos nodos son mantenidos por los llamados mineros (si bien reciben también otros nombres tal y como veremos posteriormente), los cuales cobran una comisión para mantener la red operativa. Los mineros son ordenadores que aportan poder computacional en la red para verificar las transacciones llevadas a cabo.

- Red entre pares o P2P (Peer-to-Peer). Se trata de una red de nodos conectados directamente entre ellos.

- Protocolo estándar. Tiene forma de software informático y permite que una red de ordenadores (o nodos) puedan comunicarse entre sí. Existen protocolos muy conocidos, como TCP/IP 
en el caso de Internet o SMPT en el caso de los correos electrónicos. El protocolo de blockchain funciona del mismo modo: otorga un estándar común para definir la comunicación entre los ordenadores participantes en la red.

- Sistema descentralizado. A diferencia de un sistema centralizado, donde toda la información está controlada por una única entidad, en un sistema descentralizado son todos los ordenadores conectados los que controlan la red, siendo todos ellos iguales entre sí. Por lo tanto y en general, no hay una jerarquía entre los nodos, como mínimo en una blockchain pública (en el caso de una privada o híbrida sí que puede haber cierta jerarquía). La descentralización permite optimizar los costes del proceso de manera notable, pues no existe la figura de un tercero que proporcione confianza a las operaciones.

En definitiva, una blockchain es un conjunto de ordenadores o servidores llamados nodos que, conectados en red P2P, utilizan un mismo sistema de comunicación o protocolo para validar y almacenar la misma información, de forma que ésta no esté centralizada.

\subsubsection{Propiedades de una blockchain}

La tecnología blockchain se compone de tres partes que, combinadas e integradas entre sí, transfieren a la estructura anterior la propiedad fundamental de garantizar la irreversibilidad de la información:

- Criptografía. Es un procedimiento que, empleando un algoritmo con clave de cifrado, transforma un mensaje sin atender a su estructura lingüística o significado, de tal manera que sea incomprensible 0, como mínimo, difícil de comprender, a toda persona que no tenga la clave secreta de descifrado del algoritmo empleado. La criptografía tiene la responsabilidad de proveer un mecanismo infalible para la codificación segura de las redes del protocolo que rige el sistema. Es también fundamental para evitar la manipulación, hurto o introducción errónea de información en la cadena de bloques, así como la responsable de generar firmas e identidades digitales encriptadas.

- Cadena de bloques o blockchain. Es la base de datos diseñada para el almacenamiento de los registros realizados por los usuarios. Todas las blockchain tienen que actuar bajo las mismas reglas o protocolo para dar validez al bloque y a la información recogida, e incorporarlo a la cadena de bloques. Una vez realizada esta tarea, la cadena continúa con la emisión del siguiente bloque, manteniéndose inalterable la información registrada a través de la criptografía. Esta manera de funcionar elimina la necesidad de un tercer ente de confianza.

- Consenso. Se trata de una parte imprescindible entre los usuarios de blockchain. Este consenso se sustenta en un protocolo común que verifica y confirma las transacciones realizadas, y asegura la irreversibilidad de las mismas. De igual manera, este consenso tiene que proporcionar a todos los usuarios una copia inalterable y actualizada de las operaciones realizadas en la blockchain. 
En relación a los algoritmos asociados a la criptografía, estos pueden ser de diferentes tipologías:

- Algoritmo PoW (Proof of Work). Es el primer algoritmo de consenso utilizado en sistemas blockchain, siendo el más común en esta tecnología. Se basa en el poder de computación, es decir, cuanto más potencia de CPU se tiene, más posibilidades se tienen de sellar un bloque de transacciones validadas y, por lo tanto, de llevarse la recompensa. El problema de esto es que provoca que el sistema de minería sea poco descentralizado, puesto que los que tienen más poder computacional pueden ganar más y, por lo tanto, pueden invertir más al obtener las últimas máquinas específicas para la minería, de forma que los usuarios más ricos ganarán riquezas mucho más rápidamente que otros, y en ellos se centrará una gran parte de la minería. Además, se trata de un algoritmo de consenso basado en operaciones criptográficas de fuerza sucia, con una importante impronta energética. Así mismo, surge el problema de que, cuando ya no haya más monedas para minar, los mineros sólo serán recompensados por las comisiones de las transacciones. Por lo tanto, cada vez habrá menos mineros, hecho que puede llegar a que si un minero acaba teniendo un $51 \%$ o más del poder minero podrá modificar los datos de los bloques y, por lo tanto, el sistema fallará. Sería el que se conoce como el 51\% Attack. Éste sería el algoritmo de Bitcoin y Ethereum.

- Algoritmo PoS (Proof of Stake). Fue creado como alternativa al algoritmo PoW para abordar los problemas de éste. La idea fundamental es que cada minero sólo puede minar teóricamente un porcentaje de transacciones proporcional a su participación. De esta forma, si un minero poseyera el $3 \%$ de la criptomoneda, sólo podría minar un $3 \%$ de las transacciones. También tiene en cuenta la antigüedad de la posesión de las criptomonedas, de forma que cada vez que se hace una transacción esta se restablece. Así pues, con este conjunto de variables se establece quién es el encargado de sellar un bloque y quién se lleva la recompensa. En este algoritmo se habla de forjar, en lugar de minar. Cuanto mayor sea la participación de un forjador en la red, más probabilidad tendrá de ser el encargado de sellar el bloque y de llevarse la recompensa. Este algoritmo representa mejoras respecto del PoW: no hay necesidad de maquinaria costosa, puesto que un ordenador normal podrá validar bloques siempre que esté conectado en la red; mejora mucho la eficiencia energética; y permite validaciones más fieles, puesto que el hecho de sellar en función de la participación implica que el forjador es el más interesado en mantener la red segura y activa. En cuanto al riesgo del 51\% Attack, con este algoritmo el atacante tendría que acumular el $51 \%$ de la moneda para realizar el ataque, cosa que es extremadamente costoso y difícil y, en caso de que sucediera, el mismo forjador no tendría interés de atacar la red puesto que él poseería una mayoría de la moneda y esto provocaría una caída en picado de su valor. Por lo tanto, es el primer interesado en mantener la misma red segura. Éste sería el algoritmo de Nxtcoin y Peercoin.

- DPoS (Decentralized Proof of Stake). Es un sistema derivado del Pos pero más eficiente que éste, permiten a los pequeños nodos obtener cierto beneficio indirecto a pesar de no tener su equipo siempre conectado. Éste sería el algoritmo de Bitshares.

- Algoritmo Pol (Proof of Importance). No sólo recompensa a los que tienen un saldo importante a la cuenta o tienen más poder computacional, sino que también tiene en cuenta la cantidad 
de transacciones que hace el usuario y con quién las hace. Esto significa que los usuarios que ayudan activamente a la economía y, por lo tanto, que benefician a la blockchain, son lo más recompensados. Cada usuario recibe una puntuación de confianza y, cuanto mayor sea, más posibilidades hay de ser recompensado. Esto supone una distribución de la riqueza mucho más grande, de tal manera que cualquier persona que contribuya en la red podrá obtener recompensa. Éste sería el algoritmo de NEM.

- Algoritmo PoC (Proof of Cooperation). Se basa en un sistema de rotación automatizada. La generación de bloques se lleva a cabo mediante los nodos certificados de validación CVN. Estos nodos cooperan para proteger la red. Todos ellos encuentran una cantidad igual de bloques. La cooperación crea un procedimiento eficaz y completo, que facilita la seguridad, la confirmación inmediata y las microtransacciones. Para ejecutar un CVN se necesita completar un procedimiento de certificación del nodo que será gestionado por la red descentralizada de grupos locales. Combina la confianza humana y la computacional. No hay recompensa por la creación de bloques. Por lo tanto, la oferta de dinero no se cambia mediante la creación de bloques. Las tasas de transacción obligatorias van a los respectivos creadores de bloques para compensar sus esfuerzos para la ejecución de un CVN. Éste sería el algoritmo de FairCoin2.

\subsubsection{Características funcionales de las diferentes tipologías de blockchain}

Tal y como se ha comentado anteriormente, la blockchain puede ser pública, privada o híbrida, siendo una de las principales características funcionales entre las diferentes tipologías de blockchain existentes. Las primeras blockchain fueron diseñadas para cumplir con las siguientes especificaciones:

- Pública. Cualquier persona sin ser usuaria tiene que poder acceder y consultar las transacciones realizadas (permissionless).

- Abierta. Cualquier persona tiene que poder convertirse en usuaria y participar del protocolo común si posee unos mínimos conocimientos técnicos.

- Descentralizada. No existe un usuario con más poder que otro en la red, siendo todos los nodos iguales entre sí.

- Pseudoanónima. Los propietarios de las transacciones no son identificables a título personal, pero sus direcciones sí que son rastreables como consecuencia de su carácter público. Por eso la mayoría de blockchain públicas no pueden ser anónimos, a excepción de aquellas expresamente diseñadas para serlo.

Por definición, una blockchain pública es una red descentralizada de ordenadores que utilizan un protocolo común asumido por todos los usuarios y que permite a éstos registrar transacciones en el libro mayor de la base de datos. Estas anotaciones son inalterables, si bien los participantes pueden verificar de forma independiente y por consenso los cambios que se realizan en los registros. 
Las unidades de cuenta que se utilizan en las blockchains públicas a menudo son denominadas tokens. Un token es una serie de dígitos que representa un registro dentro de la cadena de bloques. Por lo tanto, un token en una blockchain pública puede ser cualquier cadena alfanumérica que represente un registro en la base de datos descentralizada y que sea aceptada, por consenso, dentro de aquella misma blockchain.

No obstante, la propia tecnología blockchain ofrece la posibilidad de establecer una cadena de bloques con otras características diferentes: privada, cerrada y con participantes identificados; privada, abierta y anónima; o bien híbrida. Uno de los argumentos empleados en sectores regulados para el desarrollo de blockchain privadas ha sido la imposibilidad de compartir, por razones regulatorias 0 de confidencialidad, sus bases de datos abiertamente. Es por este motivo que las blockchain privadas suelen ser:

- Privada. No todos los datos inscritos tienen difusión pública y solamente los participantes 0 usuarios pueden acceder y consultar todas o algunas de las transacciones llevadas a cabo (permissioned).

- Cerrada. Solamente las personas 0 entidades invitadas a participar adquieren la condición de usuarios o registradores de las transacciones. En este sentido, el protocolo predeterminado podrá incluir diferentes niveles de acceso a los usuarios, de forma que unos puedan tener la capacidad de registrar información y otros tener vetada esta opción. El diseño es siempre función de los objetivos perseguidos.

- Distribuida. El número de nodos de los que se compone puede estar limitado al número de participantes 0 a cierto número de ellos. En cualquier caso, todos los nodos se conocen entre sí. A diferencia de una blockchain pública, donde el mantenimiento de los nodos depende de la voluntad de los usuarios, en las privadas son los participantes quienes se comprometen a mantener la estabilidad del sistema.

- Anónima. Puede establecer el nivel de anonimato que quiera para realizar y proteger transacciones. Los usuarios que registran anotaciones pueden estar o no perfectamente identificados.

Los participantes en una blockchain privada, es decir, aquellos que han obtenido la condición de usuarios, están sujetos a un protocolo predeterminado que los podrá capacitar, según se establezca, para participar en el registro de las anotaciones y/o verificar los cambios introducidos en la cadena. En este sentido, una blockchain privada puede estar más centralizada y el número de nodos que componen la red podría limitarse al número de usuarios necesarios establecido por los promotores. En este caso, se hablaría de una base de datos conjunta gestionada por aquel grupo de usuarios, en la que las anotaciones realizadas son inalterables.

En general, las compañías que ya disponen de un modelo de negocio exitoso buscan mejorarlo recurriendo a blockchain privadas o híbridas. Las iniciativas de blockchain privadas suelen denominarse frecuentemente como DLT (Distributed Ledger Technology), es decir, Tecnología del Libro Mayor 
Distribuido. Por otro lado, las blockchain públicas están más centradas en la solución de necesidades transversales implícitas en el mundo digital, como pueden ser el comercio electrónico, la identidad digital, los medios digitales (música, vídeo e imágenes) y el propio Internet de la información.

Como se ha visto, una blockchain privada es distribuida, pues es una base de datos repartida en diferentes nodos, mientras que una pública es descentralizada, pues no se controla quién participa en la misma. Se puede afirmar que una blockchain distribuida es una blockchain masivamente descentralizada, donde prácticamente todo el mundo ejecuta un nodo. En una red centralizada hay un nodo que centraliza toda la información; en el caso de una descentralizada, hay diferentes nodos que son quienes se encargan de hacerlo; y en el caso de una distribuida, son todos y cada uno de los nodos quienes se encargan de guardar la información correspondiente.

En el ámbito de las blockchain las posiciones ideológicas pueden ser muy dispares. En un extremo se dan posicionamientos derivados del movimiento original de blockchain pública, que son extremadamente progresistas, cuando no libertarios y con propuestas inconformistas y antisistema. En el opuesto, se encuentra el mundo de las blockchain privadas, que en muchas ocasiones representan la manera más formal y tradicional de entender los negocios. Y entre unos y otros se sitúa una mirada de posturas intermedias, impregnadas de pragmatismo, que apuestan por proyectos estables en el tiempo.

\subsubsection{Usabilidad de la blockchain: el Bitcoin}

Las blockchain, ya sean públicas, privadas o híbridas, como tecnologías que son no pueden mejorar la vida de las personas por sí solas. Es más bien el uso que los seres humanos pueden hacer de ellas el que mejorará nuestras vidas, pues pueden ser usadas de muchas maneras. Por ejemplo, con la primera blockchain pública, el Bitcoin, tanto se puede favorecer la inclusión financiera en países en desarrollo y aumentar el valor recibido por los destinatarios de remesas internacionales, como se puede proceder a comprar armas bajo el anonimato.

La primera aplicación práctica de la tecnología blockchain fue la creación de la criptomoneda Bitcoin en enero del año 2009. Esta aplicación empleaba dos elementos clave: la creación de un libro de registro público descentralizado; y el protocolo que permite mover e intercambiar nuevos registros entre las partes sin la intervención de un tercero.

Una criptomoneda es una vía de pago digital basada en la criptografía, las transacciones de la cual son llevadas a cabo de manera anónima y se anotan en un libro contable distribuido o blockchain. Sus propiedades son:

- Seguridad. El uso de funciones criptográficas en las transacciones insertas en las cadenas de bloques soluciona dos de los principales problemas que aparecen en otras monedas digitales: el doble gasto, es decir, que una misma unidad o fracción de la moneda pueda ser gastada 
más de una vez; y la falsificación de la moneda, es decir, que puedan emplearse monedas no autorizadas. Una vez encriptada la transacción, junto con un indicador de tiempo e introducida en el bloque de transacciones correspondiente, los balances del emisor y el receptor se ven modificados de manera irreversible. A medida que se van añadiendo nuevos bloques con nuevas transacciones, ligadas criptográficamente a las anteriores mediante funciones criptográficas, se incrementa la seguridad de las transacciones llevadas a cabo. La irreversibilidad de las transacciones evita que se reviertan transferencias de dinero alegando que no se autorizó la transacción, incluso cuando se llevó a cabo la transacción, tanto del dinero como de los bienes o servicios.

- Descentralización. Las transacciones se añaden, bloque a bloque, en un libro contable distribuido entre múltiples equipos o nodos. A diferencia de las vías de pago centralizadas, donde se suelen anotar las operaciones en un servidor único (o con copias de seguridad en diferentes servidores distribuidos), en la blockchain se incorporan en primer lugar en uno de los equipos participantes o nodo y, posteriormente, se va transmitiendo la nueva cadena al resto de nodos conectados en el sistema. Cada nueva transmisión es llamada confirmación. A mayor número de nodos, mayor es la dificultad de poder modificar la cadena de bloques y, por lo tanto, más difícil es que un agente externo modifique las transacciones. Cuando una de las copias observa una alteración de la cadena, esta copia es rechazada por el resto de nodos. Por lo tanto, a mayor descentralización, mayor será la inalterabilidad de las transacciones llevadas a cabo. Esta es una de las características más atractivas de las criptomonedas, pues no es necesaria la existencia de autoridades centrales ni de intermediarios que validen y autoricen las transacciones que se realizan en el sistema. Así mismo, la velocidad de confirmación es mayor que en los sistemas convencionales, en los que siempre existe un tiempo durante el cual se puede rechazar la transacción llevada a cabo.

- Anonimato. Esta característica viene dada por el hecho que para participar en los intercambios no es necesario llevar a cabo una identificación exhaustiva ni ceder múltiples datos privados, sino que es suficiente disponer de una cartera o wallet en la que se mantengan las criptomonedas, y desde la cual se emitan y se reciban. Con un rastreo de las operaciones llevadas a cabo, sumado a otras características (como la IP del ordenador o el origen de las primeras criptomonedas que se incluyeron en el wallet, o las obligaciones legales de identificación que tienen que realizarse a la hora de cambiar estas criptomonedas por moneda de curso oficial a través de los exchanges o casas de cambio), se podría identificar el emisor o el receptor de las monedas.

Según el trabajo titulado "Criptomonedas de los bancos centrales", de Bech y Garrat (2017), las diferentes tipologías de dinero se pueden clasificar en función de cuatro características: la accesibilidad universal, la modalidad electrónica, la emisión por parte de un banco central y la modalidad par a par o P2P. Según esta clasificación, el dinero en metálico es universalmente accesible, emitido por un banco central y par a par. Las cuentas corrientes bancarias son universalmente accesibles, electrónicas y emitidas por un banco central. En el caso de las criptomonedas, serían universalmente accesibles, electrónicas y par a par. Así pues y según esta clasificación, las criptomonedas salen del ámbito 
de emisión y control de los bancos centrales y, por lo tanto, de los Estados, y se convierten en una tipología de dinero con potencialidad propia e independiente.

Tal y como desarrollan Lara y Demmler (2018) al comparar las criptomonedas con el dinero fiduciario, se observan características diferenciales en las primeras: descentralizadas, pues no están controladas por ningún Estado, banco o institución financiera; anónimas, pues permiten preservar la privacidad al llevar a cabo transacciones; internacionales, pues pueden utilizarse en cualquier país del mundo por igual; seguras, pues las monedas no pueden ser intervenidas por nadie; sin intermediarios, pues las transacciones se realizan directamente de persona a persona; más rápidas, pues las transacciones son mucho más rápidas que las transacciones a través de entidades financieras; y con un uso voluntario, pues no es impuesto por la fuerza tal y como sucede con las monedas de curso oficial.

\subsection{Implicaciones socioeconómicas y barreras de la tecnología blockchain}

La blockchain representa una base tecnológica que puede cambiar tanto la estructura organizativa de la sociedad como los mecanismos de gobierno aplicados. Como por ejemplo, el paso de una sociedad oligárquica como la actual a una de descentralizada, con organizaciones que no tengan agentes intermediarios, neutrales y que se basen en la colaboración de todos sus miembros.

La tecnología blockchain no tan sólo es una tecnología innovadora, sino también disruptiva, dando poder a las sociedades en algo tan natural e incontrolable como es la economía. Las sociedades tienen la opción de utilizar y difundir estas tecnologías y de exigir a las autoridades la devolución de los principios de confianza, transparencia y descentralización. Las tecnologías disruptivas están llamadas a causar un impacto en los sistemas tradicionales en beneficio de los ciudadanos, obligando a una reivindicación de los obsoletos e ineficientes esquemas actuales.

La blockchain ofrece una oportunidad de dar autenticidad a lo que se entiende por colaborativo respecto a las propuestas que existen a día de hoy. Sus propiedades de descentralización permiten que el valor del ecosistema no sea acumulado en una compañía o marca central, sino que el valor final generado sea justamente distribuido entre los participantes que hayan aportado un valor inicial a la comunidad.

Sin embargo, las principales barreras que presenta esta tecnología tienen relación con las implicaciones que ésta tiene ante el posible cambio del sistema socioeconómico vigente: proporciona libertad de acción a los individuos ante los oligopolios, las grandes corporaciones y los Estados; el intercambio libre de criptomonedas implica la no necesitado de bancos; la privacidad de las transacciones económicas implica la dificultad de la aplicación de impuestos por parte de los Estados; y el registro global y universal, sin que nadie lo pueda manipular, implica que todas las transacciones queden almacenadas por siempre y, por lo tanto, que no tenga cabida el derecho al olvido o a una segunda oportunidad. 


\section{4. ¿Son compatibles la Economía Social y Solidaria y la tecnología blockchain?}

Una vez presentada la contextualización de la Economía Social y Solidaria y su integración dentro del sistema socioeconómico vigente, así como la tecnología blockchain con las características y tipologías actualmente existentes, se analiza a continuación si comparten valores, principios y objetivos o si, por el contrario, son del todo incompatibles entre sí.

Tal y como se muestra en la llustración 2, para hacerlo se analiza la compatibilidad existente entre los cuatro niveles en que se estratifica la tecnología blockchain (la estructura que la conforma, las propiedades de las que dispone, las características funcionales de las diferentes tipologías existentes, y la usabilidad que se le puede dar) y los tres ejes vertebradores y transformadores de la Economía Social y Solidaria (la gestión democrática y participativa, la orientación a las necesidades humanas y el compromiso con el entorno).

\section{llustración 2. Compatibilidad entre los ejes vertebradores de la Economía Social y Solidaria y los niveles de estratificación de la tecnología blockchain}

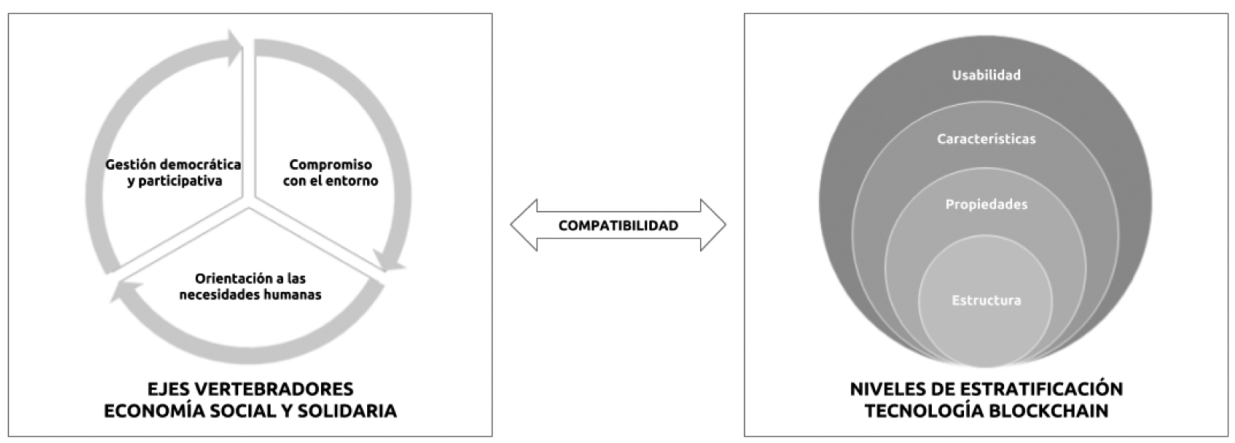

FUENTE: Elaboración propia.

Tal y como se ha comentado anteriormente en relación a la estructura de la tecnología blockchain, es un conjunto de ordenadores o servidores llamados nodos que, conectados en red P2P, utilizan un mismo sistema de comunicación o protocolo para validar y almacenar la misma información, de forma que ésta no esté centralizada. Si bien los cuatro elementos que componen la estructura propia de blockchain (los nodos, la red P2P, el protocolo estándar y el sistema descentralizado) son neu- 
tros por sí mismos, el trasfondo perseguido con la estructura resultante puede considerarse que se alinea totalmente con el eje vertebrador de la Economía Social y Solidaria correspondiente a la gestión democrática y participativa. En cuanto a los otros dos ejes, el de la orientación a las necesidades humanas y el del compromiso con el entorno, no hay criterios suficientes como para valorar si se alinea 0 no, pues hay matices sobre los que no se puede concretar, como por ejemplo: la sostenibilidad ambiental, que depende de las características funcionales de la blockchain y no de su propia estructura; y la satisfacción de las necesidades humanas o el fomento de acciones en pro de la comunidad, que dependen de la usabilidad de la blockchain y no de la estructura.

En relación con las propiedades de la blockchain, la combinación e integración de las tres partes de las que se compone una blockchain (criptografía, cadena de bloques y consenso) le transfieren a la estructura anterior la propiedad fundamental de garantizar la irreversibilidad de la información. A priori esta irreversibilidad es neutra, es decir, no proporciona criterios suficientes como para poder valorar la alineación o no con los tres ejes vertebradores de la Economía Social y Solidaria. Para poder valorarlo, se tiene que profundizar en el conocimiento de la tipología de algoritmos empleados en la parte criptográfica. Estos algoritmos pueden basarse en pruebas de trabajo (PoW), de participación (PoS-DPoS), de importancia (Pol) o de cooperación (PoC), entre otros. En función de la prueba empleada, la huella ecológica y el espíritu colaborativo y colectivista serán más o menos importantes. Así por ejemplo, la prueba de trabajo (PoW) requiere de una fuerza de computación tan elevada que genera un fuerte impacto ambiental, al mismo tiempo que fomenta una desigualdad cada vez más creciente entre las personas y la competitividad entre ellas. En el otro extremo se encuentra la prueba de cooperación (PoC), con un consumo energético mínimo y con el fomento de la participación y la colaboración entre las personas. Así pues, la alineación con los ejes vertebradores de la Economía Social y Solidaria desde el punto de vista de las propiedades de las que dispone una blockchain, dependerá sobre todo de la tipología de algoritmos empleados en la parte criptográfica.

Respecto a las características de la tecnología y tal y como se ha analizado anteriormente, la blockchain puede ser pública (abierta, descentralizada y pseudoanónima), privada (cerrada, distribuida y anónima) o híbrida (una configuración combinada de las dos anteriores). La blockchain pública tiene un modelo organizativo que no es jerárquico. En el caso de la blockchain privada o híbrida, pueden presentar cierta jerarquía y son totalmente distribuidas. Así pues, en función de si la blockchain es pública, privada o híbrida, el control, la gestión y la decisión sobre la información y el valor estará en mayor o menor grado en manos de los usuarios de la red. Es decir, una blockchain pública cumplirá mucho mejor con la gestión democrática y participativa que una privada o híbrida, que puede ser que cumpla o no en función de su grado de jerarquización. Por tanto, en función de si la blockchain es pública, privada o híbrida, se tendrán indicios sobre si su posicionamiento es más o menos transformador o conservador y, por lo tanto, si está más o menos alineada con los ejes vertebradores de la Economía Social y Solidaria.

Por último y en relación con la usabilidad de la blockchain, destacar que como tecnología es neutra. El que le da una determinada orientación es la decisión humana de emplearla con fines más 0 
menos éticos. En función de éstos, la tecnología blockchain estará orientada a necesidades sociales y ambientales, a necesidades meramente materialistas, 0 a necesidades con una configuración combinada de las dos anteriores. Tal y como se ejemplificaba en apartados anteriores, con el Bitcoin, tanto se puede favorecer la inclusión financiera en países en desarrollo y aumentar el valor recibido por los destinatarios de remesas internacionales, como se puede proceder a comprar armas bajo el anonimato. Por tanto, la alineación con los ejes vertebradores de la Economía Social y Solidaria, desde el punto de vista de la usabilidad que se le puede dar a una blockchain, dependerá de los objetivos finalmente perseguidos con su uso, más allá que esta tenga como elemento clave el almacenamiento de registros digitales, el intercambio de activos digitales o tokens, o el uso general y la ejecución de contratos inteligentes.

En este sentido, en la Tabla 1 muestra la relación existente entre los cuatro niveles de la tecnología blockchain y los ejes vertebradores de la Economía Social y Solidaria. Tal y como se observa, la mera existencia de la tecnología blockchain no garantiza la consolidación del rol transformador que ofrece la Economía Social y Solidaria, ya que no es vinculante de por sí.

\section{Tabla 1. Relación entre los niveles de la tecnología blockchain y los ejes de la Economía Social y Solidaria}

\begin{tabular}{|l|c|c|c|c|}
\hline $\begin{array}{l}\text { Blockchain } \\
\text { Economía Social y Solidaria }\end{array}$ & Estructura & Propiedades & Características & Usabilidad \\
\hline Gestión participativa & Vinculante & No vinculante & No vinculante \\
Compromiso con el entorno & No vinculante & No vinculante & No vinculante \\
Orientación a necesidades humanas & No vinculante & No vinculante & No vinculante & $\begin{array}{l}\text { No vinculante } \\
\text { No vinculante }\end{array}$ \\
\hline
\end{tabular}

FUENTE: Elaboración propia.

\section{Conclusiones: posibilidades para un nuevo paradigma social}

En base al análisis realizado sobre la relación existente entre los cuatro niveles en que se estratifica la tecnología blockchain y los tres ejes vertebradores y transformadores de la Economía Social y Solidaria, se puede concluir que ambos ámbitos serán compatibles siempre y cuando las características de la tecnología blockchain empleada se alineen con los principios de la Economía Social y Solidaria. Si es así, esta tecnología representará realmente una herramienta que permitirá ayudar al fortalecimiento y la consolidación del rol transformador que presenta la Economía Social y Solidaria 
y, por lo tanto, en la creación de un futuro tanto eficiente como equitativo, basado en los valores de la cooperación, la colaboración, la compartición, el consenso y la confianza.

En la medida que las tecnologías blockchain sean públicas (o privadas con una estructuración no jerárquica), con usos sociales y ambientales (o económicos, siempre y cuando sean respetuosos con la sostenibilidad), con algoritmos basados en pruebas que minimicen la huella ecológica, con libertad de acceso (o con un acceso restringido minimizado) y con un posicionamiento mucho más transformador que no conservador, estas tecnologías se alinearán en mayor medida con los ejes vertebradores y transformadores de la Economía Social y Solidaria: la gestión democrática y participativa, la orientación a las necesidades humanas, y el compromiso con el entorno.

Las mejoras que esta tecnología puede proporcionar en temas clave como la gestión global de las aportaciones económicas superando las actuales ineficiencias, así como la transparencia con el objetivo de evitar comportamientos fraudulentos, sitúan a la blockchain como una alternativa real y factible para mejorar la gestión de las entidades de Economía Social y Solidaria. No obstante, el propio carácter disruptivo de la propia tecnología blockchain en relación al sistema socioeconómico vigente será el que, en función de la reacción de éste hacia la transformación y el cambio, permitirá que la ayuda de la blockchain al fortalecimiento y la consolidación de la Economía Social y Solidaria sea de mayor o menor envergadura. En definitiva, es un tema sobre el que se debe continuar investigando con el objetivo de hacer realidad su acercamiento regulando formas de cooperación reales, factibles y duraderas.

\section{Bibliografía y referencias consultadas}

BECH, M. \& GARRAT, R. (2017): Criptomonedas de los bancos centrales, Banco de Pagos Internacionales.

BELZUNCES, M. (2017): "Blockchain: què és i com ens canviarà la vida", página web VilaWeb. Catalunya.

BRETOS, I. \& MORANDEIRA, J. (2016): "La economía social ante la actual crisis económica en la comunidad autónoma del País Vasco", REVESCO, Revista de Estudios Cooperativos, 122, 7-33.

CABEZAS, J. (2016): Blockchain: el futuro ya no es lo que era, Asociación de Antiguos Alumnos del Centro de Estudios Financieros.

CAÑIGUERAL, A. (2016): "Cap a una economia colllaborativa «responsable»", Revista Oikonomics, $n^{\circ} 6$. 
CHAVES, R. \& MONZÓN, J.L. (2018): "La economía social ante los paradigmas económicos emergentes: innovación social, economía colaborativa, economía circular, responsabilidad social empresarial, economía del bien común, empresa social y economía solidaria", CIRIEC-España, Revista de Economía Pública, Social y Cooperativa, 93, 5-50, DOI: 10.7203/CIRIEC-E.93.12901.

CHAVES, R. \& ZIMMER, A. (2017): El Tercer Sector en España y en Europa: crisis y resilencia, Fundació General de la Universitat de València, Àrea de Cooperació i Publicacions de la Universitat de València, La Nau Solidària.

CORRONS, A., FUSTER, M. \& ESPELT, R. (2017): Economia Social i Solidària a l'Àrea Metropolitana de Barcelona: contextualització i situació actual, Agència de Desenvolupament Econòmic, Barcelona.

DELOITTE (2017): Blockchain: economía de confianza. Tomando el control de la identidad digital, Tech Trends 2017 - The kinetic Enterprise.

FERNÁNDEZ, A. \& MIRÓ, I. (2016): L'Economia Social i Solidària a Barcelona. Les altres economies s'obren pas a la ciutat, Comissionat d'Economia Cooperativa, Social i Solidària - Ajuntament de Barcelona.

FUSTER, M., SUBIRATS, J., BERLINGUER, M., MARTíNEZ, R. \& SALCEDO, J. (2016): Procomún digital y cultura libre. ¿Hacia un cambio de época?, Editorial Icaria.

GARCÍA, J. (2016): "L'economia del futur ha de ser social i solidària", Revista Oikonomics, nº 6.

GARCÍA, J. (2017): L'economia solidària en 100 paraules, Editorial Icaria.

GÓMEZ, D. (2017): Transitant cap al procomú: La Comunificadora, Barcelona Activa.

GÓMEZ, V. \& GÓMEZ-ÁLVAREZ, R. (2016): "La economía del bien común y la economía social y solidaria, ¿son teorías complementarias?", CIRIEC-España, Revista de Economía Pública, Social y Cooperativa, 87, 257-294, DOI: 10.7203/CIRIEC-E.87.8807.

LARA, G. \& DEMMLER, M. (2018): "Social Currencies and Cryptocurrencies: Characteristics, Risks and Comparative Analysis", CIRIEC-España, Revista de Economía Pública, Social y Cooperativa, 93, 265-291, DOI: 10.7203/CIRIEC-E.93.10978.

MONZÓN, J.L. (2006): "Economía Social y conceptos afines: fronteras borrosas y ambigüedades conceptuales del Tercer Sector", CIRIEC-España, Revista de Economía Pública, Social y Cooperativa, 56, 9-24.

PREUKSCHAT, A. (2017): Blockchain: la revolución industrial de Internet, Ediciones Gestión 2000, Grupo Planeta.

PREUKSCHAT, A. (2017): "Blockchain para reinventar el funcionamiento de las relaciones humanas", El Economista, España. 
PREUKSCHAT, A. (2017): "Criptomonedas y tokens no son el mismo criptoactivo", El Economista, España.

PREUKSCHAT, A. (2017): "El impacto de Blockchain en la mejora de las vidas de las personas", El Economista, España.

PREUKSCHAT, A. (coord.) (2018): Comunidad Blockchain. El futuro de la criptoeconomía descentralizada y las ICO's, Blockchain España.

SURINACH, R. (2016): Les Altres Economies de la ciutat. Identificant l'ecosistema d'economies transformadores de Barcelona, Comissionat d'Economia Cooperativa, Social i Solidària i Consum de l'Ajuntament de Barcelona i Barcelona Activa. Editorial Montaber.

SURINACH, R. (2017): Economies transformadores de Barcelona. Comissionat d'Economia Cooperativa, Social i Solidària i Consum de l'Ajuntament de Barcelona, Editorial Marge Books.

TAPSCOTT, D. \& TAPSCOTT, A. (2017): La revolución blockchain: Descubre cómo esta nueva tecnología transformará la economía global, Editorial Deusto.

TEBBENS, W. (2016): Polítiques municipals en l'àmbit de la Societat del Coneixement i la Ciutat Intel.ligent. Cap a un model més sostenible, social i democràtic, Free Knowledge Institute. 
